Article

\title{
On Non-Linear Behavior of Viscosity in Low-Concentration Solutions and Aggregate Structures
}

\author{
Vasilis K. Oikonomou 1,2,3 \\ 1 Department of Physics, Aristotle University of Thessaloniki, 54124 Thessaloniki, Greece; \\ v.k.oikonomou1979@gmail.com or voikonomou@auth.gr or voiko@physics.auth.gr \\ 2 Laboratory of Theoretical Cosmology, Tomsk State University of Control Systems and Radioelectronics \\ (TUSUR), 634050 Tomsk, Russia \\ 3 Department of Physics, Tomsk State Pedagogical University, 634061 Tomsk, Russia
}

Received: 17 July 2018; Accepted: 24 August 2018; Published: 31 August 2018

\begin{abstract}
In this paper, an experimental method that may reveal possible aggregate symmetrical structures in highly diluted solutions is proposed, generated by the method of the release activity, which is not yet completely proven. The release activity phenomenon (regardless of whether or not it is real) could be viewed as being quite controversial. However, the focus of this paper is to reveal any possible higher-order, pragmatic, underlying symmetry or structure supporting this theory, by proposing an experiment based on viscosity. Our proposal is based on the sequential measurement of the viscosity of a highly diluted solution and the perturbative expansion of the viscosity as a function of the concentration. The coefficients of this perturbative expansion directly quantify the modification of the hydrodynamic flow around particles and around higher-order structures. Any deviation from a linear dependence of the viscosity, as a function of the concentration, could potentially reveal a collective structure of some sort, or some symmetrical pattern in the solvent. We describe our experimental proposal for non-electrolyte solutes, and future directions for revealing collective structures in solutions are discussed as related to the release activity method. Regardless of whether or not the release activity is pragmatic, it needs to be scrutinized in order to reveal its inner workings. Finally, some theoretical arguments are presented to support the proposal.
\end{abstract}

Keywords: viscosity; high dilution; release activity; virial coefficients for solutions

\section{Introduction}

In modern medicine, it is important to have effective drugs at dosages that do not cause side-effects (i.e., to minimize their toxicity for the human body). This task is of great importance for two reasons: first and most importantly, it ensures the health of patients, and secondly it makes the drugs more effective at lower cost. In this context, the release activity drugs [1-8] may offer the possibility of having more effective drugs with less side-effects on the human body. In the last 20 years, several experimental studies have been performed on release activity drugs, and it seems that they have a notable impact on patients, with minimized side-effects [2]. In fact, there seems to be a counterintuitive phenomenon (i.e., the release activity) behind the effect of these drugs. The release activity drugs are generated by many sequential dilutions of a solution, in which the initial substance has a very small concentration at the end of the process in comparison to the initial solution. This release activity phenomenon is claimed to be conceptually similar to the homeopathic method of creating drugs by its supporters, but is completely different. It is also claimed to be different from the water memory effect, since many experiments support the release activity phenomenon [2]. In all the experiments performed to date (e.g., [2] and references therein), there seems to be an underlying pattern behind the release activity phenomenon that is yet to be 
understood. However, the release activity phenomenon is quite counterintuitive, and to some extent can be viewed as controversial. Therefore, any regular pattern that can support or even disprove this theory should be carefully examined.

The release activity method is based on multiple consecutive reductions of the concentration of the parent substance, which seems to have a direct effect on the parent substance solution, altering the spatial structure of the parent substance [2]. In effect, the chemical, physical, and biological properties of the initial solution seem to be changed. Indications show the formation of supramolecular matrices which combine body molecules into semantic molecular assemblies [9]. Therefore, the underlying pattern behind the release activity method seems to indicate that there is some sort of collective physical effect underlying the release activity phenomenon. This collective effect could be formed by all the molecules of the highly diluted solution of the substance.

The drugs created based on the release activity have a common property of exerting a direct modifying biological effect on the spatial structure of the initial substance, and induce conformational changes in the initial substance, altering to some measurable extent the intrinsic physical, chemical, and biological properties. This effect is very specific and exclusively directed at the initial substance or structurally similar molecules. In effect, release activity drugs are targeted chemical substances, meaning that they have a targeted effect, not a global effect on the human organism. This means that these drugs affect the molecules they were intended to affect, not possibly structurally similar molecules. In effect, these qualify as biological products and not homeopathic drugs, although the latter are also based on the high dilution method. An example of the targeted effect of release activity drugs is offered by AS-100, which covers some effects of diazepam. From the experiments, it is known [9] that AS-100 demonstrates anxiolytic and nootropic activities integrating neutrophic and neuroprotective functionalities in a single remedy. However, AS-100 has no muscle-relaxing effect. So, a natural conclusion is that AS-100 mainly affects the central nervous system. In contrast, diazepam also affects the autonomous nervous system, since it also remedies tetanic muscle spasms at doses of $5-10 \mathrm{mg}$. The muscle spasms of tetanus are quite severe (approximately 0.05 Amperes), in contrast to the normal maximum current of spasms during an exercise, which is approximately 0.01 Amperes. Due to the muscle-relaxing effects, it is obvious that diazepam possibly acts in the ganglia of the autonomous nervous system. Therefore, it seems that AS-100 is quite targeted.

Release activity drugs are generated by diluting the initial substance to low concentrations, but clearly it is not a dose of a drug of the initial substance. Hence, homeopathic or ultralow terminology does not apply.

The release activity phenomenon is a counterintuitive phenomenon, as has already been mentioned, and it seems that the decrease in the concentration of the initial substance is a complicated process that has no direct analogue in nature. We need to note that the initial substance does not disappear completely from the solution after sequential decreases in its concentration, but the release activity effect seems to modify the whole chemical and physical structure of the solution-the properties of which do not seem to depend on the presence of molecules of the initial chemical substance in the resulting highly diluted solution.

In view of the above features of release activity drugs, it seems that a collective phenomenon is underlying the release activity, so it is vital to reveal its physical properties (if it is pragmatic, of course). In this paper, the aim is to propose an experiment that may possibly reveal the presence of a collective structure in a highly diluted solution. We are interested in connecting the physical properties of a non-electrolyte solution to the concentration of a highly diluted solution. The fundamental property to be considered is the solution viscosity, in which the solvent is disturbed by the presence of a solute at high dilution. It is important to note that the solution must not be an electrolyte, in order to avoid complicated electrostatic structures (e.g., electrostatic complexes). In this context, the flow around a single molecule of the solute in the solution is modified due to the presence of the solute molecule, and due to the interaction with the particle structure of the solution. So, is there a way to tell in a solute-independent way whether there exists a collective phenomenon that could indicate how release 
activity works? According to the experiment that is proposed in this work, the answer might be yes, since our method is based on a perturbation expansion of the viscosity in terms of the concentration, and this method works only for highly diluted solutions. Note that our proposal does not prove the release activity, but can set a solid physical basis which will provide insights towards the collective phenomenon that may underlie the release activity, if any. Additionally, the drugs that the method presented in this work may be applied to are mainly liquid drugs in aqueous solution. In principle, other drugs may also be taken into account, but the experiments presented in this work are based on homogeneous solutions.

\section{Concentration Dependence of Viscosity in Highly Diluted Solutions: An Experiment Proposal}

As is already mentioned in the introduction, if the release activity phenomenon is a pragmatically manifested physical phenomenon, it will completely change the way of thinking since it represents a breakthrough in the context of standard physicochemical processes. Since the release activity seems to be related to some underlying collective pattern, this underlying structure must manifest itself in a physical way. In fact, the chemical and biological manifestations of this underlying collective structure are already studied and confirmed experimentally. So what now remains is to check the physical manifestations of this structure. This task is crucial, and it will be the first step towards modelling this unconventional physical phenomenon.

Our proposal is related to measurements of the viscosity of various non-electrolyte solutions, as a function of the concentration, at low concentrations. Several studies exist in this line of research—see $[10,11]$ and references therein-mainly focusing on polymers or other macromolecules. Similar works can be found in [12,13]. We will base our analysis closely on Refs. [10,11] and the notation used in these references is adopted. An empirical perturbation expansion for the concentration dependence of the viscosity of a solution, is the following [10,11],

$$
\eta=\eta_{0}\left(1+a_{1} c+a_{2} c^{2}+a_{3} c^{3}+\ldots+\text { Higher order terms }\right),
$$

where $\eta_{0}$ is the viscosity of the pure solvent and $c$ denotes the concentration of the solution. It is important to stress two features of the expansion (1): firstly, it is a perturbation expansion, so this expansion holds true only in a highly diluted solution; secondly, the soluble must not be an electrolyte. Both the conditions suit the study of release activity, since the high dilution technique is the core of the release activity method, and secondly, if the soluble is a non-electrolyte, this would mean that first-order electrostatic effects can be neglected, meaning that no chemical complexes of electrostatic nature can be formed. The latter is very important since evidence for a collective molecular effect in the solvent at low concentrations is investigated, so the initial substance plays a modifying role during the process of dilution, but at low concentrations, the direct electrostatic effects, like the ones forming electrostatic chemical complexes, must be absent.

The coefficients $a_{i}, i=1,2,3 \ldots$ are important to the analysis, and the determination of these may eventually indicate the presence of an underlying collective molecular structure. Let us discuss the physical significance of these coefficients. The expansion (1) is an empirical relation that actually models the hydrodynamic flow of the solution around each molecule of the initial substance. It is of crucial importance to note that some model for the shape of the molecule of the initial substance must be assumed, and in most studies, a spherical shape, or a dumbbell shape is assumed, and the above empirical relation holds approximately true. In the chemical literature, considerable efforts have been devoted to the calculation of the coefficients $a_{i}, i=1,2,3 \ldots$. Let us discuss what these coefficients are, and their significance. The first coefficient is very important; it is called intrinsic viscosity, and it may be present for various shapes of molecules. In this paper, it is assumed that the particles have a spherical shape, for simplicity, since the intrinsic viscosity is crucially affected by the shape of the molecules of the solute. Thus, the simplest case is considered in this paper. Albert Einstein was the first to use expansions such as Equation (1), and he originally assumed a spherical shape for the molecules [14]. 
The intrinsic viscosity can be defined in terms of the relative viscosity, and it is actually the limit of the reduced viscosity as the concentration approaches to zero. The relative viscosity is equal to

$$
\eta_{r}=\frac{\eta}{\eta_{s}}
$$

where $\eta_{r}$ is the relative viscosity, $\eta$ is the viscosity of the soluble, and $\eta_{s}$ is the viscosity of the solvent. Additionally, the relative viscosity of a solution is,

$$
\eta_{s p}=\eta_{r}-1
$$

Returning to the significance of $a_{1}$, this term measures the disturbance of the hydrodynamical flow of the solvent, caused by the presence of solute particles at high dilution and distance between them. Notice that this term appears in the linear power of the concentration. The idea behind the presence of a collective structure underlying the release activity can be seen even at this stage. What we have at hand is a highly diluted solution, so a measurement of a large coefficient $a_{1}$ can already indicate the presence of an underlying physicochemical structure, unknown for the moment. This would indeed be the case, if even at low concentrations, one observes large hydrodynamic disturbances in the solution, even at nearly zero concentrations. It is also very important to note that the presence of large molecular structures may affect, to some extent, the hydrodynamic flow around each molecule, so at first, only substances with a small molecular weight must be used. So at a first step, the experiment proposed in this work has the following steps:

- Choose a substance with small molecular weight

- Choose a solvent with known viscosity at a given temperature (The temperature might affect the viscosity, but this is discussed later on.)

- Determine experimentally the viscosity-concentration dependence.

- Repeat the experiment for a number of various substances and solvents.

If a large coefficient $a_{1}$ is determined through the experiments, there must be some collective first-order underlying structure, meaning that the structure affects the molecule-molecule hydrodynamic flow of the solvent. For example, if the experiments indicate a linear dependence of the viscosity as a function of the concentration, this indicates that only the $a_{1}$ term would be important, and also the coefficient $a_{1}$ can easily be determined by the graph $\eta-c$.

More importantly, let us proceed to higher-order terms in the perturbation expansion. Theoretically, the calculation of the coefficients $a_{2}, a_{3}$ and so on, is a highly non-trivial task and a theoretical challenge. The experimental and empirical determination of these may offer insights for higher-order collective structures. The coefficient $a_{2}$ measures the modification of the hydrodynamic flow around a soluble particle caused by the presence of a second particle at some finite distance. Accordingly, the coefficient $a_{3}$ indicates the modification of hydrodynamic flows caused by higher-order molecular structures, such as molecular complexes of non-electrolytes.

Our proposal now becomes transparent: If the experiment for the determination of the function $\eta(c)$ indicates some polynomial form, this would clearly indicate the presence of some underlying higher-order spatial structure, that causes the disturbance of the hydrodynamic flow. This, in effect, would work favorably towards the release activity phenomenon, since the measured effect corresponds to extremely low concentrations. Hence, this would provide a hint of the physical manifestation of the release activity. In the literature, there exist empirical relations for the coefficients $a_{2}, a_{3}$, etc., which are $[10,11]$,

$$
a_{2}=k_{1} a_{1}^{2}, a_{3}=k_{2} a_{1}^{3}, \ldots,
$$

so the coefficients $a_{i}, i=2,3, \ldots$ quantify the second- and higher-order aggregation effects manifested in the hydrodynamic flow. Of course, these are empirical relations, so they should be explicitly checked for each substance that is tested for release activity aggregate phenomena. For polymers, 
the coefficients $k_{i}, i=1,2, \ldots$ do not depend on the molecular size and they usually vary for different soluble-solvent systems. The determination of relations similar to the ones appearing in Equation (4) may offer insights for the solutions used in the release activity drugs; however, what is needed in order to indirectly confirm the presence of aggregate effects, is to find large coefficients $a_{i}$.

Let us use a different expansion from that of Equation (1), and assume that the following perturbation expansion for a non-electrolyte highly diluted solution holds true [11],

$$
\left.\frac{\eta_{s p}}{c}=[\eta]+a_{2} c+a_{3} c^{2}+\ldots . .+ \text { Higher order terms }\right),
$$

where in this case, the intrinsic viscosity is denoted as $[\eta]$, and $\eta_{s p}$ is defined in Equation (3). The perturbation expansion (5) is believed to be empirically valid for a large number of non-electrolyte solutions, at high dilution. In this case too, regularities of the form (4) are expected [10,11],

$$
a_{2}=k_{1}[\eta]^{2}, a_{3}=k_{2}[\eta]^{3}, \ldots,
$$

and the numbers $k_{i}$ have the same significance as in the previous case, and are characteristic of the substance-solvent system. Essentially, the parameter $a_{2}$ indicates how the patterns of the hydrodynamic flow of each individual molecule of the parent substance are affected by the presence of another molecule at large distance. The empirical relation (5) is valid only at small concentrations, so this perturbation expansion would break down at higher concentrations. Clearly, the presence of the linear term $\sim a_{2} c$ would indicate the appearance of only one aggregation effect, that of molecule-molecule type; however, if for the release activity phenomena, some collective spatial structure is underlying the system of solvent-solute, then we believe that there is a possibility that this aggregation phenomenon is manifested by the appearance of higher-order terms in the expansion (5). This would imply the presence of higher-order terms and not only of the first term $a_{2}$. Consider, in particular, an aggregation molecular structure formed in space in the solution. The hydrodynamic interaction between these higher-order aggregation structures will modify the hydrodynamic flow of the soluble, and these effects would manifest their presence by contributing higher-order terms in the perturbative expansion of Equation (5). This is basically our proposal of finding direct physical manifestations of the release activity phenomenon. It is important to stress some constraints that must be satisfied. Particularly, it is of vital importance to find substances with small molecular weight. This is due to the fact that in order for the perturbative expansion (5) to hold true, the dimensions of the molecules must be much smaller than the intermolecular distances, with the latter being proportional to $\sim(M / c)^{1 / 3}$, where $c$ is the concentration and $M$ is the molecular weight. Hence, since at low concentrations, $c$ is extremely small, it is conceivable that only substances with small molecular weight qualify to be used in the experiment. Additionally, it is assumed that the orientation of particles axes is random, and all interactions between groups of each molecule are neglected. This is why long molecular structures should be avoided, so only small molecular weight molecules should be used. Finally, it is assumed that the molecules are uniformly distributed in space. This is also supported by the release activity phenomenon methodology, since it is vital to shake the solution before any experiment is performed, in order to make the solution as homogenized as possible. To sum up, the proposed experiment comprises the following steps:

- Find an appropriately selected initial substance with small molecular weight.

- Find an appropriate solute, and determine its viscosity before the substance is added to it.

- Add the substance to the soluble and shake the solution in order to obtain uniformly distributed molecules of any kind, as much as possible.

- $\quad$ Find the viscosity of the solution after the substance is added to the soluble.

- Perform measurements for various concentrations, by sequentially diluting the concentration. Start with the initial concentration and continue until a high dilution is achieved. 
- Determine any regularities at each step of each dilution. These regularities would be empirical relations between the coefficients $a_{2}, a_{3}$ and so on, for example $a_{2}=k_{1}[\eta]^{2}, a_{3}=k_{2}[\eta]^{3}$, not necessarily of this functional form. Any form of the regularities is of importance, since this may indicate the actual form of the underlying aggregate structure (if any).

- At low concentrations, is any deviation from the linear function $\frac{\eta_{s p}}{c}=[\eta]+a_{2} c$ observed? If yes, what is the form of the curve $\frac{\eta_{s p}}{c}-c$ ? This provides the clue. If the release activity phenomenon drastically affects the solvent, then it is possible to observe higher-order effects quantified in the curve $\frac{\eta_{s p}}{c}-c$.

- The experiment should be carried out a number of times in order to see the validity of the results and minimize the possibility of errors.

- $\quad$ Perform the experiment at various temperatures. Is there any notable feature observed?

- Perform the experiment with other substances and repeat the above procedure.

- Is there any regularity or pattern that indicates some collective aggregation effect?

- It is optimal to perform the experiment with non-release activity-related substances. What should be observed in this case? If release activity is a realistic phenomenon in nature, then this should be manifested in other substances too, if these qualify and satisfy the constraints imposed above. However, if there is a particular pattern for certain substances that the release activity phenomenon triggers, this should be reported. Perhaps this feature could drastically change the up-to-date formal way of thinking in physical chemistry.

With regard to the sequential dilution of the solution mentioned above, this is required in order to keep the homogeneity of the solution at all steps. If the dilution is sequential, then the changes of the solution are performed at a slow rate, and possible aggregate structures may form. It is analogous to adiabatic processes in thermodynamics; every step must be done slowly so that the system is at an equilibrium state. In the solution case, the equilibrium could be viewed as the state of having a homogeneous solution.

Additionally, it is important to note that in this paper the concentration-dependent viscosity curves are studied and examined without taking into account other important factors, such as $\mathrm{pH}$ and the temperature. Some of these factors are taken into account in the literature; see, for example [13], so a more sophisticated approach should also take into account the above factors.

Before closing, there are two important remarks which need to be stressed. In the above steps, we included two features which we did not discuss earlier, namely the intrinsic viscosity and the temperature. With regard to the former, it is of vital importance, in our opinion, to measure the viscosity of the solvent before the substance is added, and compare it to the viscosity after the substance is added. Moreover, it is important to measure the viscosity after a high number of dilutions. Even if a small difference is observed from the initial value of the solvent viscosity before the substance was added, this is important and the cause of this peculiarity should be further scrutinized. This could also qualify, in a moderate way, as indirect proof of a release activity aggregation phenomenon directly in the solvent. With regard to the temperature, knowing how the viscosity behaves as a function of the temperature may reveal, in principle, the ways that heat transfer is generated in the diluted solution. Again, a comparison of the solute viscosity before the substance is added, and after a high number of dilutions, might be of some importance, since this would also indicate that some physical property, such as heat transfer, is affected by the dilution of the substance. If one of the aforementioned effects takes place in the experiments proposed above, this could be an interesting starting point for revealing the inner workings of a highly counterintuitive phenomenon, that of release activity. The possibilities for further studies could be abundant, and many new questions arise, but all the aforementioned tests must be performed. As is already mentioned, the above would just be a starting point, not proof of release activity-related physical phenomena. 
Finally, it should be highlighted that the existence of non-linearities in the concentration dependence of viscosity has also been pointed out in previous works [10,11,13,15-17]. The main aim of this article is to embed the above theoretical frameworks in the context of release activity and related phenomena, thus offering an alternative viewpoint on this newly discovered phenomenon, by using already known results that may apply in this case. Thus, with the experiment proposed above in detail, some features of the release activity phenomenon may be proved.

\section{Theoretical Arguments on Non-Linear Viscosity-Concentration Dependence}

In the previous section, an experimental method which may reveal non-linearities in the function $\eta(c)$ was discussed and proposed, which may reveal the behavior of the viscosity as a function of the concentration. In this section, some theoretical arguments already existing in the literature [17] are presented, which may support the proposal of this paper. In fact, it has been known for quite some time that aggregates in highly diluted solutions may change the functional behavior of the viscosity as a function of the concentration [17]. In fact, the generation of aggregates completely changes the rheological and some physical properties of highly diluted solutions, introducing a new fundamental length in the theory, called the fractal dimension " $D$ ". These non-linear effects are strange to perceive, since in most solutions, the various properties are determined using the additive contribution of each particle constituting the solution. Hence, the viscosity as a function of the concentration has the form $\eta \sim c$, hence it is a linear function. If, however, aggregate structures of any form are formed in the solution, then the relation $\eta \sim c$ no longer holds true. In fact, it is quite challenging to determine the function $\eta(c)$, since this depends on various factors, such as the shape of the aggregate structures and so on. However, the generation of aggregates and the non-linear function $\eta(c)$ is expected to be a universal feature in some highly diluted solutions. In the case of having no aggregates, the viscosity as a function of the concentration is given below,

$$
\eta \sim \eta_{0} v_{0} c
$$

where $\eta_{0}$ is the intrinsic viscosity of the solution, $v_{0}$ is the average volume of each particle in the solution and $c$ the concentration of particles. If aggregate structures of any shape are formed in the solution, then the above Equation (7) no longer holds true, since the contribution of each particle in the viscosity is negligible. Only the aggregate structures contribute to the viscosity now. Consider an aggregate structure consisting of $n$ individual particles, with the volume of the structure being $v_{0}$. The density of the aggregate is [17],

$$
v(n)=v_{0} n^{3 / D},
$$

and in effect, the viscosity is [17],

$$
\eta \sim \eta_{0} v(n) u(n),
$$

where $u(n)$ is the number density of clusters with $n$ particles and $D$ is the fractal dimension. The function $u(n)$ is model dependent, and under some simple assumptions made for the aggregates-such as that the size distribution of the clusters is in a steady state and the rates for the coalescence of cluster aggregates are constant—one has [17],

$$
\eta \sim \eta_{0} v_{0}(p / q)^{(3-D) / 2 D}\left(c / m_{0}\right)^{(3+D) / 2 D},
$$

where $m_{0}$ is the mass of each particle. It is conceivable that Equation (10) is an approximation, so one may use the experimental method of the previous section in order to see whether the above equation can be fitted by experimental data. However, the inverse method should be correct, that is, obtain a number of experimental data for various solutions, and then determine which theoretical description fits better the data. In future works, we hope to address this highly non-trivial issue. 


\section{Conclusions}

The release activity phenomenon is undoubtedly intriguing and challenges all conventional scientific approaches. There are indications that this phenomenon is pragmatic. These indications come from diverse scientific methodologies, but no real proof exists to date. There exist many candidate theories that can theoretically harbor this release activity phenomenon: it may be due to particle retention in the surface monolayer, a proposal which relies on air bubble complexes, or possibly due to the presence of nanobubbles, which are nanometric superstructures; it is possibly explained by the formation of long-lived submillimeter density inhomogeneities. The most appealing, from our perspective, and the most fruitful from an experimental perspective, is the explanation based on the long dissipative water structures. According to this theory, the release activity is due to the effect of various symmetries formed in the solvent caused by the effect of the original added substance in the solution. These aggregate structures may interact in a different way, each time, with the target used for the remedy. There are, for example, indications of chiral superstructures formed in water solutions surrounding DNA molecules, which were reported in [15]. There are also reports on formations of nanoassociates in highly diluted aqueous solutions [16], which may also be a possible answer to the release activity phenomenon. Finally, the generation of aggregates as in Ref. [17], may explain the release activity phenomenon.

In this paper, an experiment is proposed that may reveal aggregate effects on solutions that are being highly diluted, by measuring the viscosity of the solution as a function of the concentration. Our proposal towards finding hints of the release activity phenomenon is based on, and actually works only on, highly diluted solutions of substances with small molecular weight. Our method will provide hints of the release activity phenomenon and cannot be viewed as direct proof. However, the existence of aggregate-collective structures in highly diluted solutions is something important by itself and can be a strong indication that the release activity phenomenon is a pragmatic phenomenon with realistic manifestations in terms of physically measurable quantities, such as the viscosity. Of course, there are many questions to be answered, the most important being, is this phenomenon generic to all substances, or does it only occur to some? With regard to the latter question, extremely low concentration experiments of a certain class of antibiotics related to DNA molecules strongly indicate regularities that were disregarded at the time the experiment was performed. We will report on this issue soon, with a new experiment focused on highly diluted solutions of antibiotics, of the order $10^{-14}$ moles/L. The path towards obtaining the exact physical underlying theory of the release activity phenomenon may be long, but it is worthwhile since this procedure will lead to less toxic drugs than the current biochemical procedures offer. However, solid experimental and theoretical work must pave the way towards describing this phenomenon, if it is a pragmatic phenomenon.

One issue that is not appropriately addressed in this introductory paper is the effect of 3-dimensional structures on the viscosity, which may be related to non-native aggregation effects, which in turn might play a role in increasing the viscosity of the solutions. This issue is very important, but was not addressed in this work for simplicity.

Funding: This research received no external funding.

Acknowledgments: This work is supported by project Physical Aspects of Release Activity, TSPU, Tomsk, Russian Federation (V.K. Oikonomou).

Conflicts of Interest: The authors declare no conflicts of interest.

\section{References}

1. Epstein, O.I. Reliz-Aktivnost' (Sovremennyy Vzglyad na Gomeopatiyu i Negomeopatiyu); Publishing House of the Russian Academy of Medical Sciences: Moscow, Russia, 2017; 47p.

2. Epstein, O. The Spatial Homeostasis Hypothesis. Symmetry 2018, 10, 103. [CrossRef]

3. Shellam, G.R.; Nossal, G.J. Mechanism of induction of immunological tolerance IV. The effects of ultra-low doses of flagellin. Immunology 1968, 14, 273-284. 
4. Davenas, E.; Poitevin, B.; Benveniste, J. Effect on mouse peritoneal macrophages of orally administered very high dilutions of silica. Eur. J. Pharmacol. 1987, 135, 313-319. [CrossRef]

5. Elia, V.; Elia, L.; Marchettini, N.; Napoli, E.; Niccoli, M.; Tiezzi, E. Physico-chemical properties of aqueous extremely diluted solutions in relation to ageing. J. Therm. Anal. Calorim. 2008, 93, 1003-1011. [CrossRef]

6. Klein, S.D.; Wolf, U. Comparison of homeopathic globules prepared from high and ultra-high dilutions of various starting materials by ultraviolet light. Complement. Ther. Med. 2016, 24, 111-117. [CrossRef]

7. Montagnier, L.; Aissa, J.; Ferris, S.; Montagnier, J.L.; Lavalleee, C. Electromagnetic signals are produced by aqueous nanostructures derived from bacterial DNA sequences. Interdiscip. Sci. Comput. Life Sci. 2009, 1, 1-90. [CrossRef]

8. Tiezzi, E. NMR evidence of a supramolecular structure of water. Ann. Chim. 2003, 93, 471-476.

9. Epstein, O.I. Release activity phenomenon and spatial homeostasis hypothesis. Uspehi Fiziologicheskih Nauk 2013, 44, 54-76.

10. Simha, R. Effect of Concentration on the Viscosity of Dilute Solutions. Trans. N. Y. Acad. Sci. 1949, 11, 409-418. [CrossRef]

11. Simha, R. The concentration dependence of viscosities in dilute solutions. J. Colloid Sci. 1950, 5, 386-392. [CrossRef]

12. Sawamura, S.; Yoshimura, Y.; Kitamura, K.; Taniguchi, Y. Effects of pressure, temperature, and concentration on the viscosity of an aqueous solution of sodium chloride. J. Phys. Chem. 1996, 13, 5526-5529. [CrossRef]

13. Kar, F.; Arslan, N. Effect of temperature and concentration on viscosity of orange peel pectin solutions and intrinsic viscosity-molecular weight relationship. Carbohydr. Polym. 1999, 40, 277-284. [CrossRef]

14. Einstein, A. Eine neue Bestimmung der Molekiildimensionen. Ann. Phys. 1906, 19, 289-306. [CrossRef]

15. Perets Ethan, A.; Yan Elsa, C.Y. The $\mathrm{H}_{2} \mathrm{O}$ Helix: The Chiral Water Superstructure Surrounding DNA. ACS Cent. Sci. 2017, 3, 683-685. [CrossRef]

16. Konovalov, A.I.; Ryzhkina, I.S. Formation of nanoassociates as a key to understanding of physicochemical and biological properties of highly dilute aqueous solutions. Russ. Chem. Bull. 2014, 63, 1-14. [CrossRef]

17. Arinshtein, A.E. Effect of aggregation processes on the viscosity of suspensions. J. Exp. Theor. Phys. 1992, 101, 1209-1212.

(c) 2018 by the author. Licensee MDPI, Basel, Switzerland. This article is an open access article distributed under the terms and conditions of the Creative Commons Attribution (CC BY) license (http://creativecommons.org/licenses/by/4.0/). 